\title{
Typical atrioventricular nodal reentrant and orthodromic atrioventricular tachycardias: electrocardiographic, electrophysiological diagnosis and treatment
}

Paroxysmal supraventricular tachycardias with narrow QRS are defined as rhythms originating from above the His bundle, heart rate higher $100 \mathrm{bpm}$ and QRS complex of less than $120 \mathrm{~ms}$ in adults or less than $90 \mathrm{~ms}$ in children. They present a prevalence of up to $8 / 1000$ individuals. The main presentations of these regular tachycardias are atrioventricular nodal re-entrant tachycardia and orthodromic atrioventricular reentrant tachycardia due to an accessory pathway. These tachycardias present morbidity, with symptoms such as palpitations, dyspnea, chest pain, syncope, polyuria, and can be a cause of sudden cardiac death. Thus, their clinical and electrocardiographic diagnoses are the first step in the approach and treatment of the patient. This review will discuss the clinical aspects, electrocardiographic, electrophysiological diagnosis and treatment options in the acute phase and long-term management, in addition to nonpharmacological treatment.

Submitted: 04 April 2016; Accepted: 23 May 2016; Published online: 28 May 2016

Keywords: Supraventricular tachyarrhythmia - Atrioventricular nodal reentrant tachycardia Wolff-Parkinson-White syndrome - Accessory pathway - Antiarrhythmic drug - Cardioversion • Catheter ablation

The narrow QRS tachycardia is that which originates above the His bundle bifurcation, with QRS complexes of less than $120 \mathrm{~ms}$ and heart rate of more than 100 beats per minute $(\mathrm{bpm})$. Its prevalence in the general population is 6 to $8 / 1000$ individuals, reaching $11 \%$ to $18 \%$ in patients with heart failure [1]. Among children the duration of the QRS of the tachycardia is equal to or less than 90 seconds and heart rate can exceed $200 \mathrm{bpm}$ in newborn infants and higher than $100 \mathrm{bpm}$ (from 130 to $300 \mathrm{bpm}$ ) in those over 10 years of age $[1,2]$.
Among the regular narrow QRS tachycardia, the most common is typical atrioventricular (AV) nodal reentrant tachycardia, followed by orthodromic AV tachycardia. Symptoms include palpitations, fatigue, chest pain, light-headedness, neck discomfort, polyuria and pre- syncope and syncope. Polyuria is due to the natriuretic peptide secreted by the distension of the atria. Syncope can occur between 15\% and $20 \%$ of patients, due to the high rate of the tachyarrhythmia ( $\geq 170 \mathrm{bpm}$ ). Besides this morbidity, these tachycardias can lead
Rose Mary Ferreira Lisboa da Silva $^{1 *}$ \& Leonardo Roever ${ }^{2}$ 'Department of Internal Medicine, Faculty of Medicine, Federal University of Minas Gerais, Brazil

${ }^{2}$ Department of Clinical Research, Faculty of Medicine, Federal University of Uberlandia, Brazil

*Author for correspondence:

Tel.: +553134099746

roselisboa@uol.com.br 
sudden cardiac arrest in $2.2 \%$ to $4.5 \%$ of patients with accessory pathways $[1,3,4]$.

Precipitating factors may be physical activity, sudden movements, and emotional stress [1,2,5-7]. In newborns and infants, paleness, lethargy, coughing, drowsiness, irritability, appetite loss, vomiting, dyspnea, cyanosis and/or diaphoresis are the symptoms of the tachycardia. Heart failure can occur if the tachycardia remains for 24 to 48 hours without treatment [8].

We searched Embase, Scielo, Europubmed, Cochrane, PubMed and CINAH databases (1990 to march 2016), using the following search strategy: (typical (All Fields) AND atrioventricular (All Fields) AND nodal (All Fields) AND reentrant (All Fields), orthodromic (All Fields) AND atrioventricular (All Fields) AND ("tachycardia”(MeSH Terms) OR "tachycardia"(All Fields) OR "tachycardias"(All Fields))). The purpose of this article is to review the clinical aspects, electrocardiographic diagnosis and treatment options in the acute phase and long-term management, in addition to non-pharmacological treatment.

\section{Typical Atrioventricular Nodal Reentrant Tachycardia}

This tachycardia is responsible for $50-60 \%$ of tachycardias narrow QRS in adults and $15 \%$ of supraventricular tachycardias in pediatric patients, predominantly from adolescence onwards. There is a female predominance, with a ratio of $2: 1$ or $3: 1$. Its manifestation occurs mainly during the third or fourth decade of life. At physical examination, "frog sign" (prominent neck pulsations) may be present during tachycardia [1-9].

The hypothesis of nodal reentry has been postulated by Mines in 1913. Goldreyer and others showed the location and the electrophysiological properties of the nodal reentry in humans, resulting in the concept of dissociation of the AV node $[9,10]$. There are dual AV pathways: the slow pathway $(\alpha)$ which transmits the electric stimuli slowly and has short refractory period, and the fast pathway $(\beta)$, with long refractory period (Figure 1). The anterosuperiors fibers that make connection between the AV node and the right atrium, near the apex of Koch's triangle, form the fast pathway. The fibers of the inferoposterior region form the slow pathway (Figure 2) [11]. Up 37\% of subjects have dual AV nodal physiology; however only a minority of these develops nodal reentrant tachycardia. Furthermore, incidence of dual AV nodal pathways decreases with ageing [12].

Electrical stimulation goes through fast pathway during sinus rhythm. When there is a supraventricular extra systole, the stimulus is conducted by the slow pathway (antegrade conduction) because it reaches earlier AV node. The retrograde conduction occurs over the fast pathway and there is almost simultaneous contraction of the atria and the ventricles. This circuit occurs in $90 \%$ of AV nodal reentrant tachycardia (AVNRT); it is the typical form [1,5,7]. Atypical or uncommon nodal reentrant tachycardia includes the fast-slow and slow-slow types and of left origin $[1,7]$.

The refractory period of the slow pathway is shorter in women, resulting in a greater difference between the fast and slow pathway refractory periods. This may explain the higher incidence of tachycardia in women [13].

\section{Electrocardiographic and Electrophysiological Diagnosis}

The electrocardiogram shows the presence of retrograde atrial activation ( $\mathrm{P}^{\prime}$ ) as pseudo $\mathrm{R}$ wave in $\mathrm{V} 1$ (Figure 3) or pseudo $S$ in the inferior leads (D2, D3 and aVF) in about $60 \%$ of cases during tachycardia [14]. Although of this criterion have high specificity (91\%$100 \%)$, it is less sensitive $(14 \%-58 \%)$ [15]. The RP' interval is equal to or less than $70 \mathrm{~ms}$ in any derivation in which to its measure. In other cases, retrograde atrial activation will be absent because it coincides with the QRS complex. When the beginning of this tachycardia is recorded, there is the increase in the PR interval. There is the presence of retrograde atrial activation, when visible, at its end. ST segment depression, and variation of the RR interval may occur during tachycardia [3,14,16-18].

Atrial pacing with extra stimuli at progressively shorter coupling intervals is used for the electrophysiological study for diagnosis of double nodal pathway. There is a prolongation of at least $50 \mathrm{~ms}$ at A-H interval (interval between the atrial electrogram and His bundle) in response to the shortening of the coupling interval of the premature atrial stimulus by $10 \mathrm{~ms}$ (defined as the 'jump-up' phenomenon). When applying atrial extra stimuli with progressively shorter coupling intervals, there is a gradual increase in A-H interval until reaching the refractory period of fast pathway. From that moment, the conduction through the AV node becomes the slow pathway. After the "jump", there is atrial echo and the beginning of tachycardia. Ventricular pacing results in induction of atypical AVNRT due to higher refractory period the fast pathway. The stimulus is conducted to the atrium by the slow pathway and it returns to the ventricle by the fast pathway $[14,18]$. The Table 1 shows the classification of AVNRT types.

\section{Treatment}

To reversal to sinus rhythm, vagal maneuver can succeed when made at the beginning of tachycardia. Over time, the maneuver is usually not effective due to sympathetic activation [19]. 


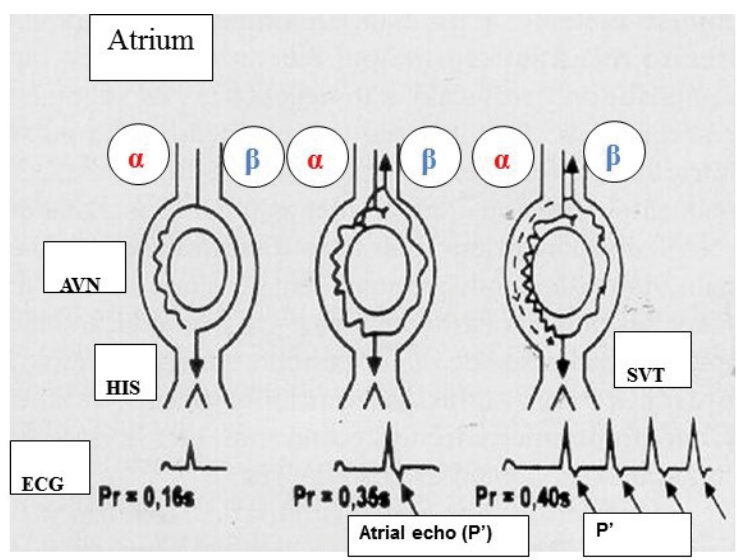

Figure 1: Representation of the typical atrioventricular nodal reentrant tachycardia circuit. a: Alpha pathway; $\beta$ : Beta pathway; AVN: Atrioventricular Node; His: His bundle; ECG: Electrocardiogram; PR: PR Interval; SVT: Supraventricular Tachycardia; P': Retrograde atrial activation.

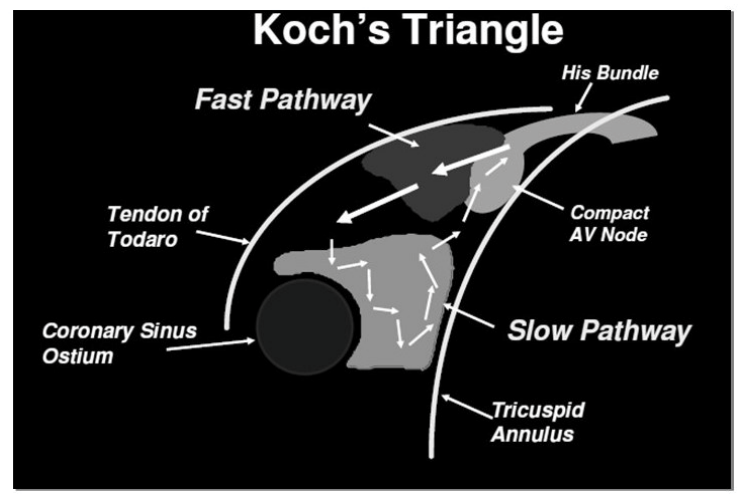

Figure 2: Representation of the triangle of Koch with its limits, compact NAV, the fast and slow tracks and bundle.

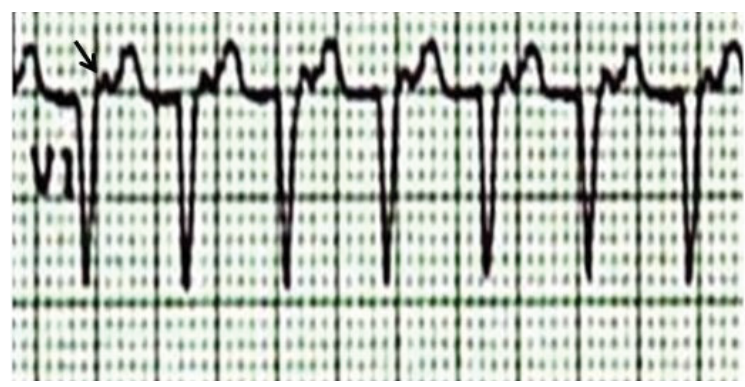

Figure 3: Electrocardiographic recording of lead V1 during the typical atrioventricular nodal reentrant tachycardia shows pseudo R (shown by arrow).

One of the vagal maneuvers is the carotid sinus massage. Its contraindications are presence of carotid bruits or significant stenosis carotid, transient ischemic accident or stroke within the past 3 months [18-20]. The main complications are neurological as dysarthria and visual disturbances and can occur in $1 \%$ of cases [21]. Carotid massage should be performed during 5 to 10 seconds and applied to the superior portion of the carotid artery in the neck with the patient in supine position. Both sides must be stimulated sequentially, starting from the right side, which has a higher success rate. Another vagal maneuver consists of applying an ice-cold wet towel to the face. Vagal maneuver with pressure in the eye should not be performed because it is harmful to the patient. The Valsalva maneuver is to increase intrathoracic pressure of $40 \mathrm{mmHg}$ for 15 seconds, by expiring against a closed glottis. The success rate of the Valsalva maneuver is $19-54 \%$ and the carotid massage is approximately $25 \%[21,22]$.

The modified Valsalva maneuver is performed immediately at the end of the strain with passive elevation to 45 degrees of the legs of the patient for 15 seconds. This maneuver is highly effective, returning more than $40 \%$ of patients to sinus rhythm compared with $17 \%$ with a standard Valsalva maneuver [23].

If these first-line interventions are not effective, the pharmacological treatment should be done with the intravenous administration of adenosine (class I) or calcium channel blocker of the phenylalkylamine (Table 2) [1,19,20,24].

Adenosine is a nucleotide with negative chronotropic and dromotropic properties with short half-life of about 10 seconds. It acts by increasing the driving of potassium channels, resulting in cell hyperpolarization and consequent reduction of electrical conduction.

Another effect is the decrease of the calcium influx due to antagonism to catecholamines. It must administered as a $0.1-0.2 \mathrm{mg} / \mathrm{kg}$ per bolus (usual maximum dose $12 \mathrm{mg}$ ) given via a central sheath or catheter and followed by a $10 \mathrm{cc}$ (cubic centimeter or milliliter) saline flush $[1,19,24]$. A lower dose $(3 \mathrm{mg})$ is recommended in persons who have a cardiac transplant [20]. Adverse effects occur in about $40 \%$ of cases, such as dyspnea, chest pain and some rhythm disturbances (asystole, bradycardia, extrasystoles, atrial fibrillation). These effects are transient; thus, the administration of adenosine is safe. The effect of the drug occurs within 15 to 30 seconds, with success reversal of $78-96 \%$. Contraindications to the use of adenosine include bronchospasm (reactive airway disease), concomitant use of dipyridamole (which inhibits adenosine metabolism) or xanthine (which is an antagonist of adenosine receptors), 2nd degree AV block or higher, sinus node dysfunction, concomitant use of verapamil and digoxin $[1,19,20,24]$.

Intravenous verapamil or diltiazem are class IIa for the reversal of tachycardia with success of 64$98 \%[1,18,19,24]$. For children under the age of 1 year, patients at risk of brady arrhythmias or with hypotension or systolic dysfunction, these drugs should not be given. Another option for reversal (Class IIa) is beta-blocker intravenously [24]. Electrical 
Table 1: Classification of AVNRT types.

\begin{tabular}{|cccc|}
\hline $\begin{array}{c}\text { Table } 1 \text { - Classification of AVNRT types } \\
\text { Typical AVNRT }\end{array}$ & AH/HA & VA (His) (ms) & Usual ERAA \\
\hline Slow-fast & $>1$ & $<60$ & His \\
\hline Atypical AVNRT & & & $>60$ \\
\hline Fast-slow & $<1$ & $>60$ & CS os/LRAS \\
\hline Slow-slow & $>1$ & CS os/LRAS \\
\hline
\end{tabular}

AH: Atrial to His interval; $\mathrm{HA}$ : His to Atrium interval; VA: interval measured from the onset of ventricular Activation on surface ECG to the earliest deflection of the atrial activation in the His bundle electrogram; ERAA: Earliest Retrograde atrial Activation; CSo: Ostium of the Coronary Sinus. LRAS: Low Right Atrial Septum.

\begin{tabular}{|c|c|c|}
\hline Class of recommendation & Intervention & Success rate \\
\hline 1 & Carotid sinus massage & $25 \%$ \\
\hline I & Valsalva maneuver & $19-54 \%$ \\
\hline I & Adenosine $0.1-0.2 \mathrm{mg} / \mathrm{kg}$ & $78-96 \%$ \\
\hline Ila & Verapamil (5-10 mg IV during $2 \mathrm{~min}$ ) or diltiazem $(0.25 \mathrm{mg} / \mathrm{kg}$ IV $2 \mathrm{~min})$ & $64-98 \%$ \\
\hline
\end{tabular}

synchronized cardioversion is indicated for patients with hemodynamic instability or if there was ineffectiveness of drugs [24].

For treatment in the chronic phase, catheter ablation (radiofrequency or cryoablation) is class I recommendation [24]. Its immediate success rate is $96-98 \%$, with risk AV block of $1.0-1.5 \%$ and late recurrence from 3\% to 7\% [19,24,25]. Among patients with AVNRT undergoing successfully ablation of the slow pathway, atrial fibrillation recurs in up to one third of them despite the decreased incidence of atrial fibrillation in patients with both arrhythmias. P wave dispersion measured by 12-lead electrocardiogram was an independent predictor of atrial fibrillation recurrence after ablation in patients with AVNRT and paroxysmal atrial fibrillation [26].

For patients who are not candidates for ablation, options of drugs are oral beta-blockers, diltiazem or verapamil $[19,24]$. Flecainide or propafenone are class IIa and sotalol, dofetilide and amiodarone are class IIb recommendation of drugs [24].

\section{Orthodromic Atrioventricular Tachycardia}

This tachycardia is the second most common among regular narrow QRS tachycardia in adults [1,7]. Among children and adolescents, it is responsible for $70-90 \%$ of narrow QRS tachycardias. Its prevalence is up to $0.5 \%$ and it is more frequent in those male with a mean age of 23 years $[1,2,5-8]$. This tachycardia is one of the signs of the Wolff- Parkinson-White syndrome described in 1930. There is abnormal pathway between atria and ventricle. The incidence of accessory pathways is 0.1 to $3.1 / 1000$ individuals, reaching $3.4 \%$ if familial incidence [14]. The preexcitation detected by the presence of delta wave on the electrocardiogram occurs between 0.15 to $0.28 \%$ in the population. However, its disappearance is observed in $22.5 \%$ of children during the follow up of 10 years. There is an association with congenital anomalies in $9-32 \%$ of cases, as the disease Ebstein, atrial and interventricular septal defects, tetralogy of Fallot, and with hypertrophic cardiomyopathy $[1,2,5-8,14,27,28]$.

The incidence of sudden cardiac death in patients with accessory pathway is between 0.15 to $0.39 \%$, reaching $3 \%$ to $4 \%$ in symptomatic young $[1,7]$. However, this can be the first demonstration in $48 \%$ of patients. The markers of risk for sudden cardiac death are presence of symptomatic tachycardia, Ebstein's disease, presence of multiple accessory pathways and RR interval less than $250 \mathrm{~ms}$ during preexcited atrial fibrillation [1,14,27-31].

The accessory pathways make the connection between the ventricular and atrial myocardium on the right or left side of heart, or with specialized cardiac conduction system $[7,14,27,28]$. Thus, there is earlier ventricular activation, with initial slurring of the upstroke of the QRS complex (delta wave) and short PR interval in classic electrocardiogram $[1,14,27,28]$. The most common location of these accessory pathways is left free wall (more than 50\%), followed by posteroseptal region (20\% to $30 \%)$, right free wall $(10 \%$ to $20 \%)$ and

septal region (5\% to $10 \%$ ) [7]. Among $2-20 \%$ of the pediatric population, accessory pathways can be multiple [7,32,33].

According to its driving property, accessory pathway is classified as manifest or concealed. Manifest accessory pathway drives electrical stimulus in antegrade conduction, i.e., from the atrium to the ventricle, with delta wave in sinus rhythm. The concealed bypass tract drives electrical stimulus only in retrograde conduction and it is present in almost half of patients with accessory pathway. However, only $5 \%$ of accessory pathways display exclusive retrograde conduction. Thus, orthodromic tachycardia occurs 
in approximately $95 \%$ of patients, with anterograde conduction of electrical stimulation over the AV node, and retrograde conduction over the accessory pathway. From $5 \%$ to $10 \%$ of patients with this syndrome can present antidromic tachycardia (wide QRS tachycardia), with reentry to the reverse sequence of electrical stimuli driving [14]. There are other rare types of tachycardia driven by an accessory pathway, such as Coumel tachycardia and Mahaim fiber-related tachycardia $[14,27,28]$.

\section{Electrocardiographic and electrophysiological diagnosis}

In sinus rhythm, electrocardiographic signals are delta wave (initial slurring of the upstroke of the QRS complex), short PR interval, when the accessory pathway is manifest, and ventricular repolarization abnormalities may be present (Figure 4) $[1,14,27,28]$. The polarity of the delta wave allows identifying the location of an accessory pathway through its vectorial orientation and morphology, R/S wave ratio and QRS polarity $[34,35]$. The preexcitation with the presence of delta wave on the electrocardiogram can be intermittent (on the same tracing there are QRS complexes with and without delta wave) due to higher refractory period of the accessory pathway. The preexcitation may be inapparent or latent, if delta wave is visible only during maneuvers or use of drugs that reduce the conduction of electrical stimuli through AV node, allowing antegrade conduction of stimuli over accessory pathway in sinus rhythm [7,14,27]. The accessory pathway is termed concealed if there is no delta wave on the electrocardiogram and its presence is documented by tachycardia or by the recording of cardiac electrograms during electrophysiological study $[5,7,14,27,28]$.

Orthodromic AV reentrant tachycardia is a narrow QRS tachycardia and RP' interval < PR interval with a slowly conducting accessory pathway, and RP' interval is greater than or equal to $70 \mathrm{~ms}$ (Figure 5) [1]. The polarity of retrograde atrial conduction ( $\mathrm{P}$ ') also allows the location of the accessory pathway.

The electrophysiological study is important for the differential diagnosis between AVNRT and AV tachycardia due to a concealed accessory pathway $[14,15]$. If there is preexcitation with delta wave, the interval measured from delta wave to His electrogram is negative, i.e., the delta wave begins prior to activation of the His bundle during sinus rhythm. Orthodromic tachycardia is triggered by spontaneous or stimulated atrial premature depolarization or ventricular extrastimulus. There is anterograde block in patients with concealed accessory pathway different from those with manifest accessory pathway. The retrograde $\mathrm{P}$ wave is not fused with the QRS or negative during tachycardia and the retrograde activation is eccentric, the opposite of what occurs in the circuit in AVNRT. The mapping with intracardiac catheters during tachycardia or ventricular pacing allows the location of the accessory pathway through the earliest retrograde atrial activation [14].

\section{Treatment}

Treatment of acute phase and long-term management are the same as described above regarding the AV nodal reentrant tachycardia $[1,19,20,24]$. Catheter ablation is class I recommendation in patients symptomatic, with success rate of $92-98 \%$ for a single accessory pathway

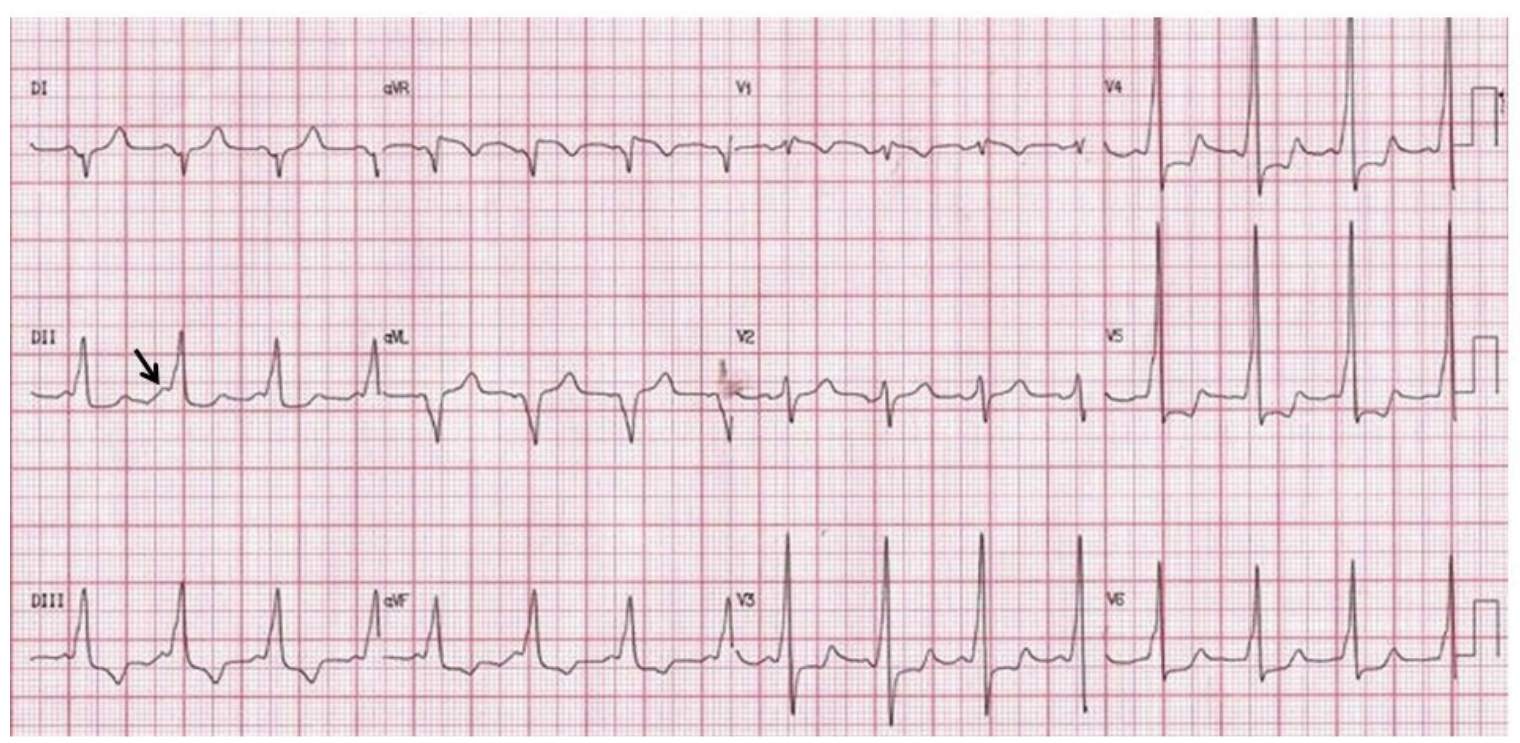

Figure 4: A 12-lead electrocardiogram during sinus rhythm with delta wave (shown by arrow), short PR interval and ventricular repolarization abnormalities, consistent with accessory pathway left location. 


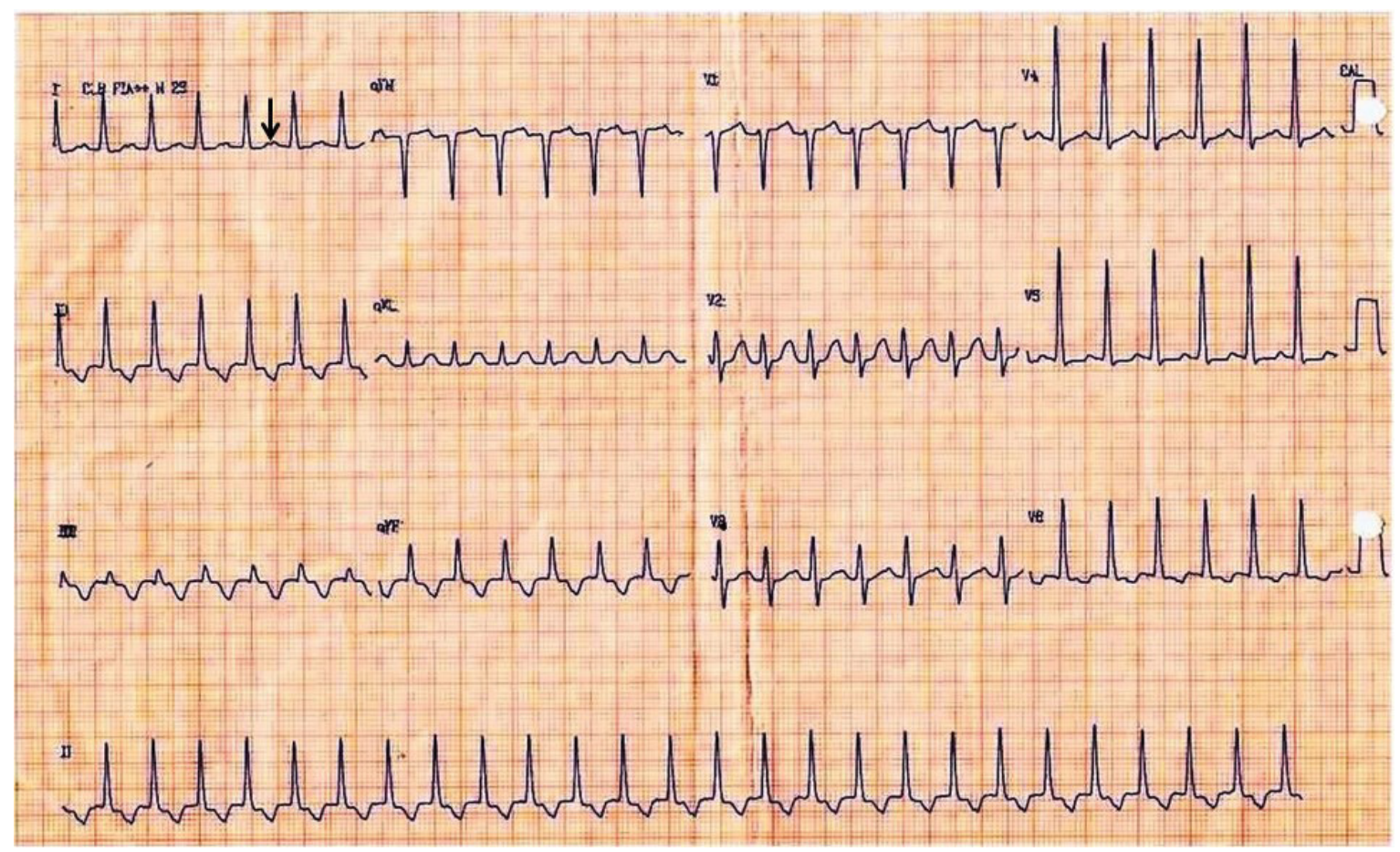

Figure 5: A 12-lead electrocardiogram demonstrated an orthodromic AV reentrant tachycardia with retrograde $\mathrm{P}$ waves (shown by arrow) and RP' interval of $160 \mathrm{~ms}$ measured at the derivation D1.

and recurrence from $2 \%$ to $11 \%$. The success rate is lower for ablation of accessory pathways on the right and the septum and its recurrence is more frequent. The ablation complications are rare (3\% of cases), such as perforation infarction, valvular lesions, coronary artery lesions, AV block, embolism and vascular complications. AV block occurs most commonly in ablation of anteroseptal or mid septal pathway [25,27].

Ablation in patients with preexcitation and asymptomatic is controversial. A systematic review showed that asymptomatic patients with accessory pathway that did not undergo ablation showed ventricular arrhythmias in $9 \%$ of cases and ventricular fibrillation in $2 \%$ of cases during follow-up [31]. Risk stratification with exercise testing or Holter is Class I recommendation [24]. Abrupt loss of delta wave during exercise or the presence of intermittent accessory pathway is indicative of low risk. Short refractory period of the accessory pathway is a predictor of severe arrhythmic events [36]. Thus, the electrophysiological study is recommended (Class IIa) for asymptomatic patients and ablation should be considered in the same procedure, according to risk stratification [24,31].

\section{Conclusion}

The most common narrow regular QRS complex tachycardias are typical atrioventricular nodal reentrant tachycardia and orthodromic atrioventricular tachycardia. The clinical presentation of these tachycardias practically does not differ from each other. The electrocardiographic diagnosis is crucial to the knowledge of its mechanism, despite similar treatment in the acute phase. Stratification of risk through noninvasive and invasive tests is important, since the incidence of sudden cardiac arrest is up to $4.5 \%$ in the Wolff- Parkinson-White syndrome. Catheter ablation is already well established. Cryoablation is an alternative energy which can reduce the risk of AV block, especially in children and adolescents. References

\section{Executive summary}

- Paroxysmal supraventricular tachycardias with narrow QRS are defined as rhythms originating from above the His bundle, heart rate higher $100 \mathrm{bpm}$ and QRS complex of less than $120 \mathrm{~ms}$ in adults or less than $90 \mathrm{~ms}$ in children.

- The main presentations of these regular tachycardias are atrioventricular nodal re-entrant tachycardia and orthodromic atrioventricular reentrant tachycardia due to an accessory pathway. 


\section{References}

1. Blomstrom-Lundqvist $\mathrm{C}$, Sheinmann MM, Aliot $\mathrm{EM}$, et al. ACC/AHA/ESC guidelines for the management of patients with supraventricular arrhythmias--executive summary: a report of the American College of Cardiology/American Heart Association Task Force on Practice Guidelines and the European Society of Cardiology Committee for Practice Guidelines (Writing Committee to Develop Guidelines for the Management of Patients With Supraventricular Arrhythmias). Circulation. 108, 1871-1909 (2003).

2. Kleinman ME, Chameides L, Schexnayder SM, et al. Part 14: Pediatric advanced life support: 2010 American Heart Association Guidelines for Cardiopulmonary Resuscitation and Emergency Cardiovascular Care. Circulation. 122, 876-908 (2010).

3. Wang YS, Scheinman MM, Chien WW, Cohen TJ, Lesh MD, et al. (1991) Patients with supraventricular tachycardia presenting with aborted sudden death: incidence, mechanism and long-term follow-up. J Am Coll Cardiol 18: 17111719.

4. Timmermans C, Smeets JL, Rodriguez LM, et al. Aborted sudden death in the Wolff-ParkinsonWhite syndrome. Am. J. Cardiol. 76, 492-494 (1995).

5. Fox DJ, Tischenko A, Krahn AD, et al. Supraventricular tachycardia: diagnosis and management. Mayo. Clin. Proc. 83, 1400-1411 (2008).

6. Brembilla-Perrot B, Marçon O, Chometon F, et al. Supraventricular tachyarrhythmia as a cause of sudden cardiac arrest. J. Interv. Card. Electrophysiol. 16, 97-104 (2006).

7. Lee KW, Badhwar N, Scheinman MM. Supraventricular tachycardia--part I. Curr. Probl. Cardiol. 33, 467-546 (2008).

8. Salerno JC, Seslar SP, Supraventricular tachycardia. Arch. Pediatr. Adolesc. Med. 163, 268-274 (2009).

9. Gürsoy S, Steurer G, Brugada J, Andries E, Brugada P. Brief report: the hemodynamic mechanism of pounding in the neck in atrioventricular nodal reentrant tachycardia. N. Engl. J. Med. 327, 772-774 (1992).

10. Scheinman MM, Yang Y. The history of AV nodal reentry. Pacing. Clin. Electrophysiol. 28, $1232-$ 1237 (2005).

11. McManus BM, Wood SM. Morphological features of normal and abnormal conduction system: essentials for electrophysiologists. In: Singer I, Barold SS, Camm AJ (eds). Nonpharmacological theraphy of arrhythmias for the 21st century: the state of the art. Armonk, New York: Futura Publishing Company, Inc. 27-56 (1998).

12. D’Este D, Bertaglia E, Zanocco A, Reimers B, Pascotto P. Electrophysiological properties of the atrioventricular node and ageing: evidence of a lower incidence of dual nodal pathways in the elderly. Europace. 3, 216-220 (2001).

13. Liuba I, Jönsson A, Säfström K, Walfridsson $H$. Gender-related differences in patients with atrioventricular nodal reentry tachycardia. Am. J. Cardiol. 97, 384-388 (2006).

14. Josephson ME. Clinical Cardiac Electrophysiology: Techniques and Interpretations. 3rd ed, Lippincott Williams \& Wilkins. 1-610 (2002).

15. Katritsis DG, Josephson ME. Differential diagnosis of regular, narrow-QRS tachycardias. Heart. Rhythm. 12, 1667-1676 (2015).

16. Arya A, Kottkamp H, Piorkowski C, et al. Differentiating atrioventricular nodal reentrant tachycardia from tachycardia via concealed accessory pathway. Am. J. Cardiol. 95, 875-878 (2005).

17. Oh S, Choi YS, Sohn DW, et al. Differential diagnosis of slow/slow atrioventricular nodal reentrant tachycardia from atrioventricular reentrant tachycardia using concealed posteroseptal accessory pathway by 12-lead electrocardiography. Pacing. Clin. Electrophysiol. 26, 2296-2300 (2003).

18. Katritsis DG, Camm AJ. Atrioventricular nodal reentrant tachycardia. Circulation. 122, 831-840 (2010).

19. Ferguson JD, DiMarco JP. Contemporary management of paroxysmal supraventricular tachycardia. Circulation. 107, 1096-1099 (2003).

20. Link MS. Clinical practice. Evaluation and initial treatment of supraventricular tachycardia. N. Engl. J. Med. 367, 1438-1448 (2012).

21. Collins NA, Higgins GL. Reconsidering the effectiveness and safety of carotid sinus massage as a therapeutic intervention in patients with supraventricular tachycardia. Am. J. Emerg. Med. 33, 807-809 (2015).

22. Smith GD, Fry MM, Taylor D, Morgans A, Cantwell K. Effectiveness of the Valsalva 
Manoeuvre for reversion of supraventricular tachycardia. Cochrane. Database. Syst. Rev. 2, CD009502 (2015).

23. Appelboam A, Reuben A, Mann C, et al. Postural modification to the standard Valsalva manoeuvre for emergency treatment of supraventricular tachycardias (REVERT): a randomised controlled trial. Lancet. 386, 1747-1753 (2015) .

24. Page RL, Joglar JA, Caldwell MA, et al. 2015 ACC/AHA/HRS Guideline for the Management of Adult Patients With Supraventricular Tachycardia: A Report of the American College of Cardiology/American Heart Association Task Force on Clinical Practice Guidelines and the Heart Rhythm Society. J. Am. Coll. Cardiol. S0735- 1097(15)05840-4 (2015).

25. Sohinki D, Obel OA. Current trends in supraventricular tachycardia management. Ochsner. J. 14, 586-595 (2014).

26. Amasyali B, Kose S, Aytemir K, et al. P wave dispersion predicts recurrence of paroxysmal atrial fibrillation in patients with atrioventricular nodal reentrant tachycardia treated with radiofrequency catheter ablation. Ann. Noninvasive. Electrocardiol. 11, 263-270 (2006).

27. Almendral J, Castellanos E, Ortiz M. Update: Arrhythmias (V). Paroxysmal supraventricular tachycardias and preexcitation syndromes. Rev. Esp. Cardiol. (Engl Ed). 65, 456-469 (2012).

28. Bhatia A, Sra J, Akhtar M. Preexcitation Syndromes. Curr. Probl. Cardiol. 41, 99-137 (2016).

29. Wackel P, Irving C, Webber S, Beerman L, Arora G. Risk stratification in Wolff-Parkinson-White syndrome: the correlation between noninvasive and invasive testing in pediatric patients. Pacing. Clin. Electrophysiol. 35, 1451-1457 (2012).

30. Pappone C, Vicedomini G, Manguso F, et al. Risk of malignant arrhythmias in initially symptomatic patients with Wolff-Parkinson-White syndrome: results of a prospective long-term electrophysiological follow-up study. Circulation. 125, 661-668 (2012).

31. Al-Khatib SM, Arshad A, Balk EM, et al. Risk Stratification for Arrhythmic Events in Patients With Asymptomatic Pre- Excitation: A Systematic Review for the 2015 ACC/AHA/HRS Guideline for the Management of Adult Patients With Supraventricular Tachycardia: A Report of the American College of Cardiology/American Heart Association Task Force on Clinical Practice Guidelines and the Heart Rhythm Society. J. Am. Coll. Cardiol. S0735-1097(15)06202-6 (2015).

32. Bromberg BI, Lindsay BD, Cain ME, Cox JL. Impact of clinical history and electrophysiologic characterization of accessory pathways on management strategies to reduce sudden death among children with Wolff-Parkinson-White syndrome. J. Am. Coll. Cardiol. 27, 690-695 (1996).

33. Zachariah JP, Walsh EP, Triedman JK, et al. Multiple accessory pathways in the young: the impact of structural heart disease. Am. Heart. J. 165: 87-92 (2013).

34. Fitzpatrick AP, Gonzales RP, Lesh MD, et al. New algorithm for the localization of accessory atrioventricular connections using a baseline electrocardiogram. J. Am. Coll. Cardiol. 23, $107-$ 116 (1994).

35. Arruda MS, McClelland JH, Wang X, et al. Development and validation of an ECG algorithm for identifying accessory pathway ablation site in Wolff-Parkinson-White syndrome. J. Cardiovasc. Electrophysiol. 9, 2-12 (1998).

36. Pappone C, Vicedomini G, Manguso F, et al. Wolff-Parkinson-White syndrome in the era of catheter ablation: insights from a registry study of 2169 patients. Circulation. 130, 811-819 (2014). 